\title{
Analysis of dynamics of a vertical cantilever in rotary coupling to the moving frame with movement limiters
}

\author{
Piotr Wolszczak ${ }^{1, a}$, Grzegorz Litak ${ }^{1,2, b}$, and Krystian Lygas ${ }^{1}$ \\ ${ }^{1}$ Faculty of Mechanical Engineering, Lublin University of Technology, Nadbystrzycka 36, Lublin 20-618, Poland \\ ${ }^{2}$ Department of Process Control, AGH University of Science and Technology, Mickiewicz Alley 30, Kraków 30-059, Poland
}

\begin{abstract}
The efficiency of the mechanical energy harvesting with the use of vibrating elements can be improved by synchronizing stimulation vibrations and own linear frequencies of systems as well as super or sub harmonics induced by non-linear phenomena. The article presents numerical cross-sectional study of the mechanical system. The system consists of an elastic beam set vertically, which the lower end is fixed in the rotary support, and is stimulated to move in the horizontal axis. The upper end of the beam is free but below its level there are bumpers limiting the free rotation of the beam. Numerical studies took into account the variability of the frequency and amplitude of the excitation beam movement, and horizontal distance between bumpers. Beam deflection was observed, on the basis of which the amount of energy generated by the piezo element was estimated. Nonlinear phenomena and analysis of frequency synchronization of vibrations improving the energy effect of an energy generator are presented.
\end{abstract}

\section{Introduction}

In the field of energy harvesting, a mechanical systems using non-linearity are used to increase the efficiency of the system with the ambient vibration sources. The frequency and amplitude of the system excitation are directly affecting the power output of the harvesting device. Account for high energy density, deflecting piezoelectric layers were proposed [1] to transduce mechanical into electrical energy. The occurrence of the nonlinear phenomena are giving additional frequency broadness of the moderate power output via inclined nonlinear resonance characteristics, multiple natural frequencies as well as their sub-harmonics and super-harmonics or dynamical hysteresis occurring with frequency change [2-4]. For industrial applications, the frequencies from over few to few hundred Hertz are of interests [5-8]. In the present note, we present the results on the bistable vibrational energy harvester with additional amplitude limiters inducing impacts $[9,10]$. Here we focus on energy efficiency with changing selected system parameters, namely: excitation parameters (amplitude and frequency), clearance between the angular amplitude limiters.

\section{Energy harvesting system}

The subject of the research was the elastic beam set in a vertical position (as in the system of the inverted pendulum) and mounted on a rotary handle. The rotating handle was placed on a trolley equipped with two stops to limit

\footnotetext{
ae-mail: p.wolszczak@pollub.pl

be-mail: g.litak@pollub.pl
}

the rotation of the beam. An important function of the bumpers is to cause collisions and increase the deflection arrow of the elastic beam. The schematic picture of the mechanical resonator is shown in Fig. 1.

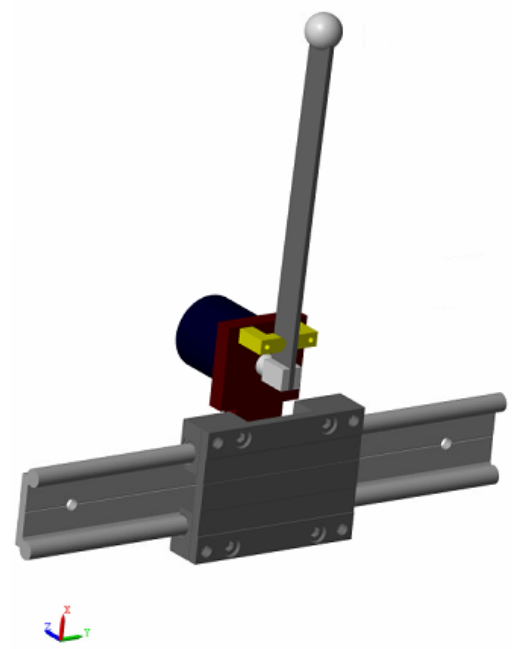

Figure 1. Schematic view of the resonator used in the Matlab program. The amplitude limiters (bumpers) are sketched in yellow. Piezoelectric layer can be placed above the contact point. Deflection of the beam will be proportional to the voltage output.

Sample time series of selected variables registered during the simulation is shown in Fig. 2. Note that at the initial conditions, the beam is in a vertical position, and then as a result of the movement of the trolley it falls onto 


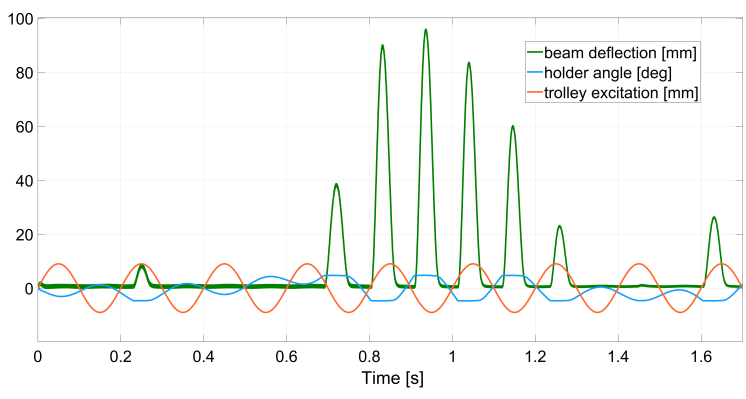

Figure 2. Sample part of the simulation results with sinusoidal excitation of the vertical beam lower end with frequency $\omega=$ $5 \mathrm{~Hz}$, amplitude $A=9 \mathrm{~mm}$ and distance between bumpers $C=10$ $\mathrm{mm}$ (where the thickness of the beam is $T=3 \mathrm{~mm}$ ).

one of the bumpers (amplitude limiters). In the further part of the simulation, the beam is stimulated by the movements of the trolley for rotary motion and collisions with bumpers. The moment when the beam again reaches the vertical position and hits the opposite bumper is achieved for a series of selected parameters. In these cases, the kinetic energy of the rotating beam must be higher than the potential energy of the beam striving for a resting position, i.e. the support on one of the bumpers [12]. Note that for $C \rightarrow 0$ we get the inverted elastic pendulum case studied by Friswell et al. [13].

For this system the physical experiments and numerical simulations (in the Matlab program using additional Simmechanics libraries) were performed after [10, 12]. During the physical experiments carried out, the beam position and deflection was observed with the high-speed camera, [9] and a piezo element was placed on the beam's wall. The voltage was recorded in the circuit equipped with the resistor and next, the amount of generated electric energy was calculated $[11,14]$. In this way, the energy efficiency of the system operation was evaluated, and the results obtained from numerical simulations were confirmed in physical experiment.

Consequently, a number of numerical simulations were performed to investigate the phenomenon of nonlinearity, evaluate the energy efficiency of the inverse beam system with buffers in the low frequency range of excitation of the beam motion.

During the simulation, the following variables were recorded: the position of the trolley, the deflection angle of the beam and the distance between the ends of the beam as a measure of beam deflection (assuming one-sided deflection, this value is proportional to arrow deflection).

A series of simulations were prepared for various sets of parameters, which were:

- amplitude of excitation of the beam in the range: $A=1$ $16[\mathrm{~mm}]$,

- frequency of beam activation in the range $\omega=1-16[\mathrm{~Hz}]$,

- distance between buffers, gap in the range $C=0-11$ [mm].

The maximum distances between the bumpers and the beam walls in the initial position were $5.5 \mathrm{~mm}, 11 \mathrm{~mm}$ in total.

\section{Results}

Fig. 3 presents the surfaces formed from the results of sum of beam deformation expressed by changing the distance between the ends of the beam [mm]) for different beam excitation frequencies. In the case of a zero distance between buffers and a beam in the charts there are minimum non-zero values. The beam then shakes and its deflection is low.

Figure 3 shows that the total deflection of the beam gets higher values as the clearance $C$ increases. Generally, energy output increases with clearance, which presumably enable the beam to accelerate just before impacts. For frequency of $1 \mathrm{~Hz}$ of excitation the influence of the clearance and excitation amplitude is the simplest and can be identified by the simple proportional relation. Increasing the frequency we observe corrugation on the resulting energy surface. It can be noticed that a similar flattened shape (with respect to $1 \mathrm{~Hz}$ results) takes the surface for an excitation frequency of $16 \mathrm{~Hz}$. This non-monotonous property against frequency focuses our range choice of $\omega$. Additionally, It can be noticed that for a higher edge of the surface for the highest value of clearance increases with the excitation amplitude, unlike $1 \mathrm{~Hz}$, where the decrease of the total beam deflection (and harvested energy output) with decreasing amplitude of the trolley for a clearance $C$ of $11 \mathrm{~mm}$ has been observed.

To illustrate the nonlinear effect, which can be noticed as the larger output deviation by changing the bifurcation parameter, on the amount of energy output we calculated deviations from the reference surface. Here the set of results by $1 \mathrm{~Hz}$ excitation was chosen as the reference surface to all other results obtained in the experiment. The simulation results for this frequency allow to achieve high values of the total deflection of the beam. The highest values were observed in the case of $2 \mathrm{~Hz}$ frequencies. Consequently, Fig. 4 presents the negative differences in the results of the total beam deflection obtained for the trolley activation frequency equal to $1 \mathrm{~Hz}$ and other systems where the excitation frequency (trolley movement) was from 2 to 16 [Hz].

On the other hand, Fig. 5 indicate positive fragments of surfaces, not visible in the figure 4 , where the differences of the total deflection of the beam to the reference surface for higher beam excitation frequencies were higher than zero.

\section{Conclusions}

We studied the energy harvesting system with the inverted elastic pendulum with amplitude limiters. Changing the excitation parameters (amplitude, frequency) and geometrical property of the system (clearance) we obtained systematic simulation results of the potential transduction efficiency. Interestingly, the clearance has a considerable effect for the whole range of the excitation frequency. It seems to be more important for the total energy output with respect to amplitude of excitation (Fig. 3). Amplitude of the excitation includes some fluctuation and the noncontinuous changes in the output energy which could 

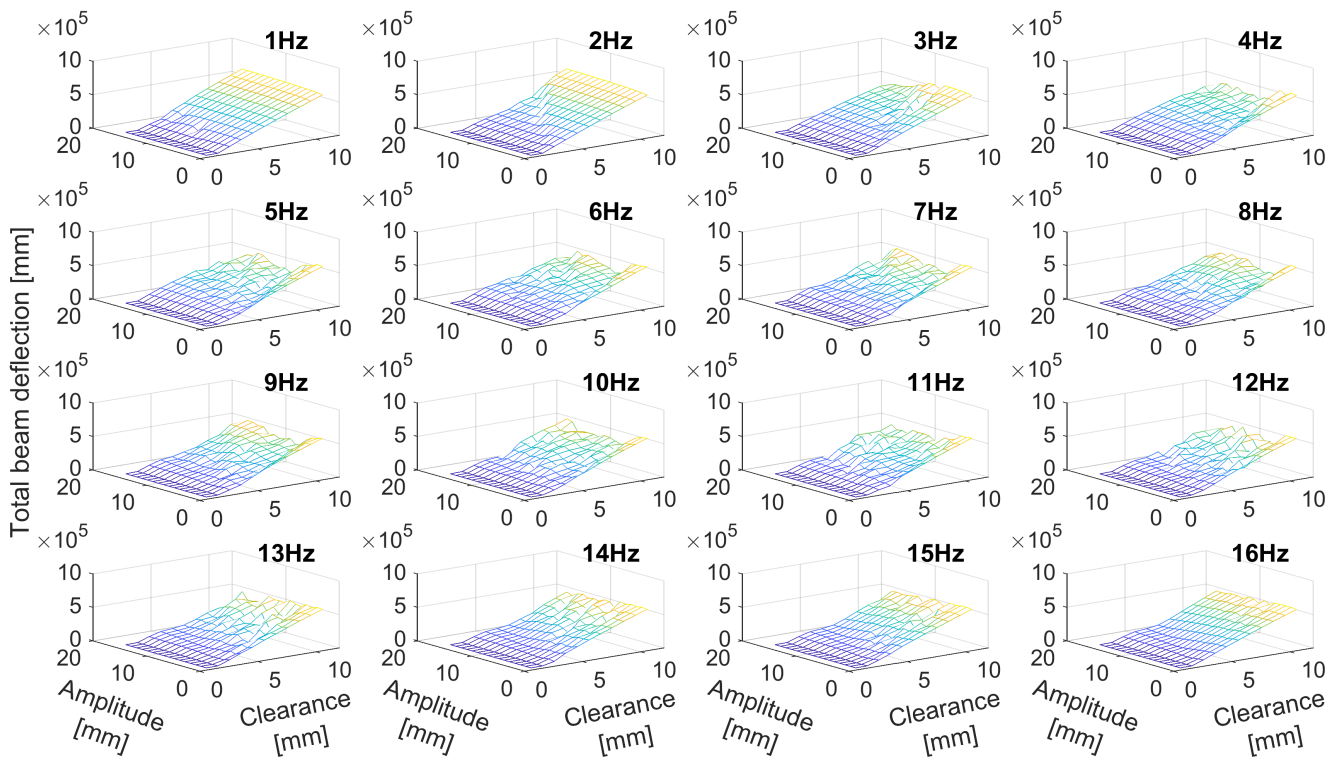

Figure 3. Surfaces formed from the results of strain distortion (sum of deflection $[\mathrm{mm}]$ ) for different excitation frequencies and amplitudes (frequency from 1 to $16[\mathrm{~Hz}]$ and amplitude from 1 to $16[\mathrm{~mm}]$ trolley) and clearance between bumpers and beam in the range from 0 up to 11 [mm]. $Z$ axis - sum of beam deflection [mm], X axis - clearance [mm], Y axis - amplitude [mm].

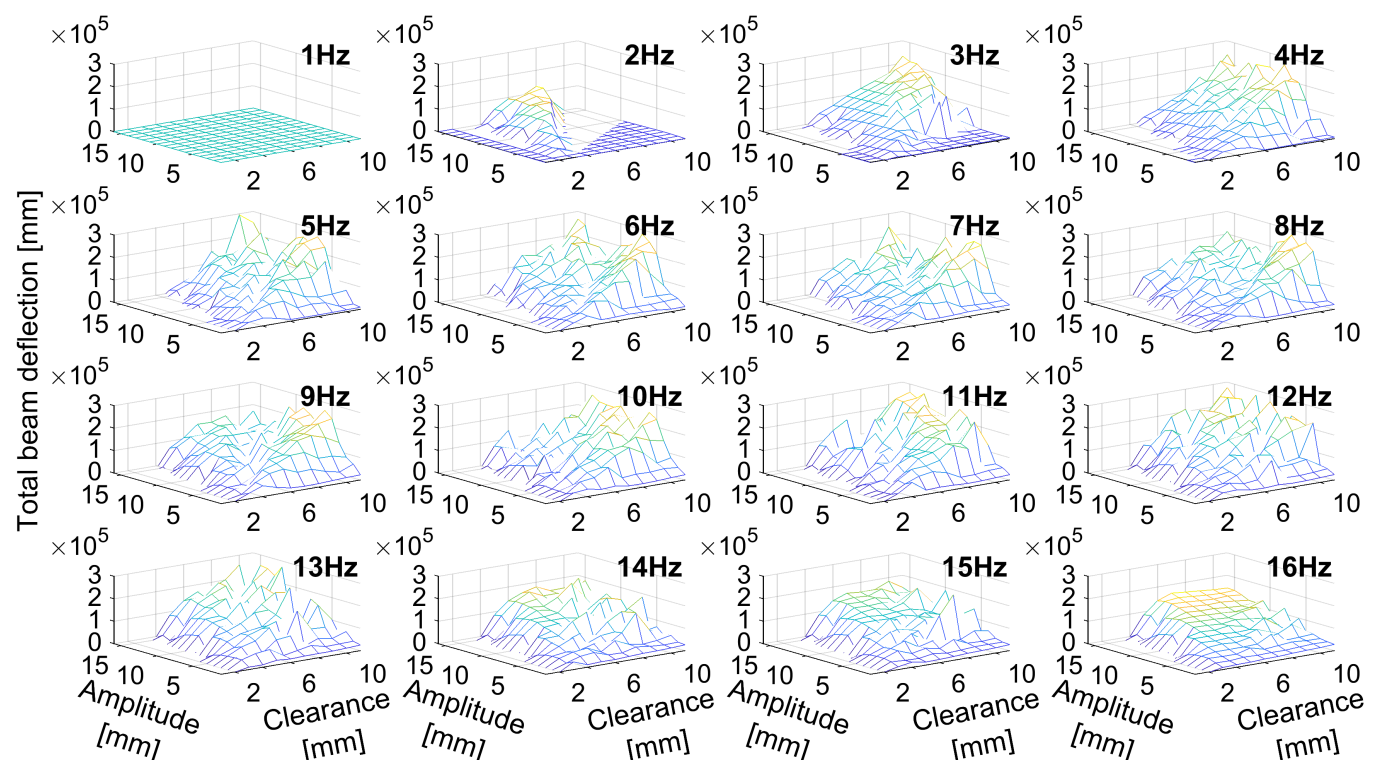

Figure 4. Differences between surfaces formed from the results of strain distortion (sum of deflection [mm]) for excitation frequencies $1 \mathrm{~Hz}$ and different excitation frequencies. $\mathrm{Z}$ axis - sum of beam deflection [mm], $\mathrm{X}$ axis - clearance [mm], $\mathrm{Y}$ axis - amplitude [mm].

reflect possible bifurcations. In the present studies we assumed only first mode solution. However, due to nonlinearities, the system was also sensitive to a super- and subharmonic resonance conditions. To complete these studies, more natural frequecies of beam oscillations should be investigated further with possibility of higher cantilever beam mode occurrence.

\section{References}

[1] S.P. Beeby, M.J. Tudor, N.M. White, Meas. Sci. Technol. 17, R175 (2006)

[2] A. Syta, G. Litak, M.I. Friswell, S. Adhikari, Eur. Phys. J. B 89, 99 (2016)

[3] T. Huguet, A. Badel, A., M. Lallart, Appl. Phys. Lett. 111, 173905 (2017)

[4] S. Zhou, J. Cao, G. Litak, J. Lin, TM-Tech. Mess. https://doi.org/10.1515/teme-2017-0076 (2018) 


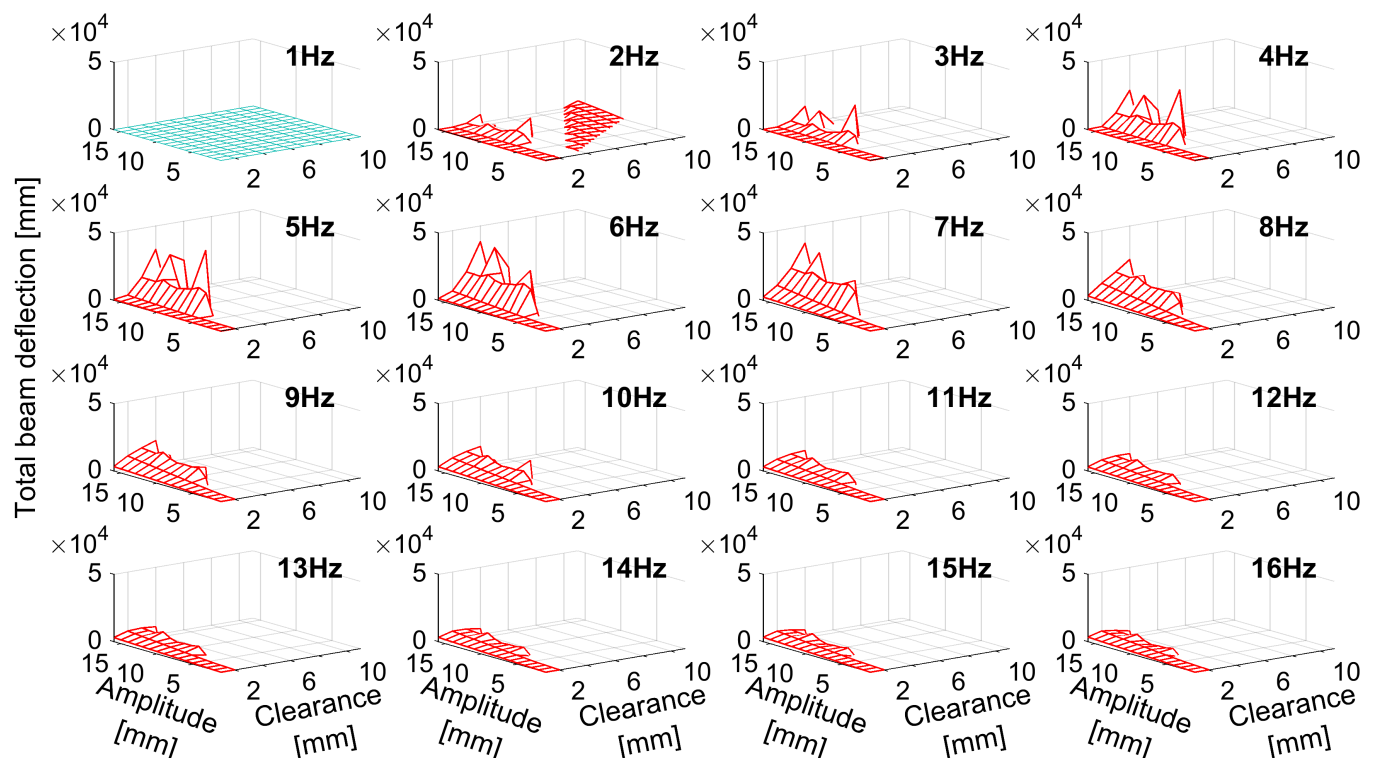

Figure 5. Fragments of surfaces of differences between results of strain distortion (sum of beam deflection [mm]) for excitation frequencies $1 \mathrm{~Hz}$ and different excitation frequencies where value were positive ( $\mathrm{Z}$ axis - sum of beam deflection [mm], $\mathrm{X}$ axis clearance [mm], Y axis - amplitude [mm]. ).

[5] P.D. Mitcheson, E.M. Yeatman, G.K. Rao, A.S. Holmes, T.C. Green, Proceedings of the IEEE 96, 1457 (2008)

[6] R.L. Harne, K.W. Wang, Smart Mater. Struct. 22 023001 (2013)

[7] S.P. Pellegrini, N. Tolou, M. Schenk, J.L. Herder, J. Intell. Mater. Syst. Struct. 241303 (2013)

[8] J. Twiefel, H. Westermann, J. Intell. Mater. Syst. Struct. 24, 1291 (2013)

[9] P. Wolszczak, K. Lygas, G. Litak, Mech. Syst. Sig. Process. 107, 43 (2018)

[10] K. Lygas, P. Wolszczak, P. Staczek, G. Litak, in Energy Harvesting for Wireless Sensor Networks: Technologies, Components and System Design Eds. O.
Kanoun and C. Viehweger (De Gruyter Oldenbourg, Berlin 2019)

[11] K. Lygas, P. Wolszczak, G. Litak, MATEC Web of Conferences 14816003 (2018)

[12] G. Litak, P. Wolszczak, K. Lygas, in Vibration, Control and Stability of Dynamical Systems. DSTA 2017, Eds. Awrejcewicz J. et al. (Department of Automation, Biomechanics and Mechatronics, ARSA, Lodz 2017) pp. 291-298.

[13] M.I. Friswell, S.F. Ali, S. Adhikari, A.W. Lees, O. Bilgen, G. Litak, J. Intell. Mater. Syst. Struct. 23, 1505 (2012)

[14] A. Koszewnik, P. Grzes, W. Walendziuk, Eur. Phys. J. Spec. Topics 224 2719, (2015) 